\title{
Adaptive Event-triggered Control over a Shared Network
}

\author{
Adam Molin and Sandra Hirche
}

\begin{abstract}
Under many circumstances event-triggered control outperforms time-triggered control schemes when resources such as communication, computation, or energy are sparse. This paper investigates another benefit of event-triggered control concerning the ability of adaptation. The system under consideration comprises multiple heterogeneous control loops that are closed over a shared communication network. Each subsystem is modelled as a discrete-time stochastic linear system. The design problem is formulated as an average cost Markov decision process (MDP) with unknown global system parameters that are to be estimated during execution. Techniques from distributed optimization and adaptive MDPs are used to develop distributed self-regulating event-triggers that adjust their transmission rate to meet a global resource constraint. Numerical simulations show the effectiveness of the approach and illustrate the convergence properties.
\end{abstract}

\section{INTRODUCTION}

The recent increased interest in networked control systems and cyber-physical systems has led to various paradigm shifts in the digital control design. The systems under consideration usually consist of a multitude of small-scale integrated entities coupled through a common communication network. The efficient usage of available resources, like communication, computation, or energy, is a prerequisite for the successful operation of such control systems. This fact has stimulated researchers to look for advanced sampling schemes beyond the conventional periodic sampling scheme to reduce resource consumption. A large amount of literature [1]-[6] shows that event-triggered control schemes achieve the same control performance as time-triggered control schemes, while reducing the number of samples significantly. Potential problem settings where event-triggered control has been applied successfully range from control over communications [1]-[4] and multi-agent systems [5] to the control design in embedded real-time systems [6].

Besides the necessity for an efficient use of resources, other non-functional requirements like self-configurability and adaptability need to be addressed within the design of networked control and cyber-physical systems. In the envisioned system, local entities, such as sensor nodes, are aware that a common resource is shared among them. Such awareness is reflected in the capability of adjusting the sampling rate adaptively to reduce resource needs, while maintaining a certain amount of performance. To be more specific, this paper considers multiple independent control

A. Molin and S. Hirche are with the Institute of Automatic Control Engineering, Technische Universität München, Arcisstraße 21, D-80290 München, Germany; http://www.lsr.ei.tum.de, adam.molinetum.de, hirche@tum.de. Research supported by the German Research Foundation (DFG) within the Priority Program SPP 1305 "Control Theory of Digitally Networked Dynamical Systems". systems, whose control loops are closed over a shared communication networks.

Related work can be found in [2], [4], [7]-[9] that analyze the performance of event-triggered schemes in contentionbased networked control systems. Conclusions vary with the choice of the communication model. Using CSMA models with priority or randomized arbitration as [2], [4], eventtriggered control outperforms significantly periodic control schemes. Unlike [2], [4], it has been shown in [7], [8] that time-triggered scheduling outperforms event-triggered schemes for slotted and unslotted ALOHA transmission schemes. In [9], time-triggered and event-triggered sampling schemes are compared within different communication models. It is concluded that the choice of both the sampling scheme and the model significantly influence the performance of the overall system.

Throughout the aforementioned work, the design of the event-triggered control scheme is determined beforehand in a centralized fashion. This might be inconvenient, as it is difficult to implement, as the number of subsystems might be large, and it lacks of flexibility, as it needs to be rerun completely whenever changes in the system occur. The ability of adaptation of event-triggered control systems has not been studied properly yet. This motivates us to address the problem of designing event-based controllers for heterogeneous subsystems closed over a common communication network in a distributed manner.

The contribution of this paper is to develop a distributed algorithm for an event-triggered control system, where each subsystem adjusts its event-triggering mechanism to optimally meet a global resource constraint imposed by the communication network. The problem is formulated in the MDP framework with an average cost criterion. Each subsystem is modelled as a discrete-time stochastic linear system with a quadratic control cost. The communication constraint is given by limiting the total average transmission rate of all subsystems. Such formulation has been successfully applied in [4] to approximate a communication system with a limited number of transmission slots per time step. Despite of the relaxation to an average transmission constraint which ignores that collisions may occur during transmission, it turns out in [4] that the approximative approach serves as a good approximation for the problem with a hard transmission constraint. Inspired by distributed optimization and adaptive MDPs, a distributed sample-path based algorithm is proposed. In contrast to [4], a dual decomposition approach related to congestion control [10] is chosen to study the underlying optimization problem. By measuring the total transmission rate, a dual price mechanism forces each sub- 
system to adjust their event-triggering thresholds. Numerical simulations are conducted to illustrate the effectiveness of the obtained algorithm.

The remaining part of this paper is structured as follows. In Section II, we introduce the system model and describe the problem statement. Section III develops the adaptive eventtriggered controller. The efficiency of the proposed approach is illustrated in Section IV by numerical simulations.

Notation. In this paper, the operator $(\cdot)^{\top}$ and $\operatorname{tr}[\cdot]$ denote the transpose and trace operator, respectively. The Euclidean norm is denoted by $\|\cdot\|_{2}$. The variable $P$ denotes the probability measure on the abstract sample space denoted by $\Omega$. The expression $F, \mathrm{P}-$ a.s. denotes that the event $F$ occurs almost surely w.r.t. probability measure $P$. The expectation operator is denoted by $\mathrm{E}[\cdot]$ and the conditional expectation is denoted by $\mathrm{E}[\cdot \mid \cdot]$. The relation $x \sim \mathcal{N}(0, X)$ denotes a Gaussian random variable with zero-mean and covariance matrix $X$. The operator $[\cdot]^{+}$denotes the projection onto the non-negative real line, i.e., $[\cdot]^{+}=\max \{0, \cdot\}$. The operator $\mathbb{1}_{\{\cdot\}}$ denotes the indicator function. The truncated sequence up to time $K$ of a signal $x_{k}, k \geq 0$, is denoted by $X_{K}$, i.e., $X_{K}=\left[x_{0}, \ldots, x_{K}\right]$. If not otherwise stated, a variable with superscript $i$ indicates that it belongs to subsystem $i$.

\section{PROBLEM STATEMENT}

Figure 1 depicts the networked control system under consideration. It shows $N$ independent control subsystems whose feedback loops are connected through a shared communication network. A control subsystem $i$ consists of a process $\mathcal{P}^{i}$, a controller $\mathcal{C}^{i}$ that is implemented at the actuator and a sensor $\mathcal{S}^{i}$. The process $\mathcal{P}^{i}$ is given by a controlled time-homogeneous Markov chain with state $x_{k}$ taking values in $\mathbb{R}^{n_{i}}$ and evolving by the following difference equation

$$
x_{k+1}^{i}=A^{i} x_{k}^{i}+B^{i} u_{k}^{i}+w_{k}^{i},
$$

where $A^{i} \in \mathbb{R}^{n_{i} \times n_{i}}, B^{i} \in \mathbb{R}^{n_{i} \times m_{i}}$. The control input $u_{k}^{i}$ is taking values in $\mathbb{R}^{m_{i}}$. The system noise $w_{k}^{i}$ takes values in $\mathbb{R}^{n_{i}}$ at each $k$ and is i.i.d. with $w_{k}^{i} \sim \mathcal{N}\left(0, W^{i}\right)$. The initial states $x_{0}^{i}, i \in\{1, \ldots, N\}$, have a distribution whose density function is symmetric around its mean value $\mathrm{E}\left[x_{0}^{i}\right]$ and has finite second moment. At each time step $k$, the scheduler situated at the sensor station $\mathcal{S}^{i}$ decides, whether a state update should be sent to the controller $\mathcal{C}^{i}$ over the contention-based network. The $i$ th scheduler is described by the variable $\delta_{k}^{i} \in\{0,1\}$, where

$$
\delta_{k}^{i}= \begin{cases}1 & \text { update } x_{k}^{i} \text { is sent } \\ 0 & \text { otherwise }\end{cases}
$$

The received signal at the $i$ th controller, $z_{k}^{i}$, is given by

$$
z_{k}^{i}= \begin{cases}x_{k}^{i} & \delta_{k}^{i}=1 \\ \emptyset & \text { otherwise }\end{cases}
$$

In order to quantify the resource constraint due to the communication network, the individual average transmission

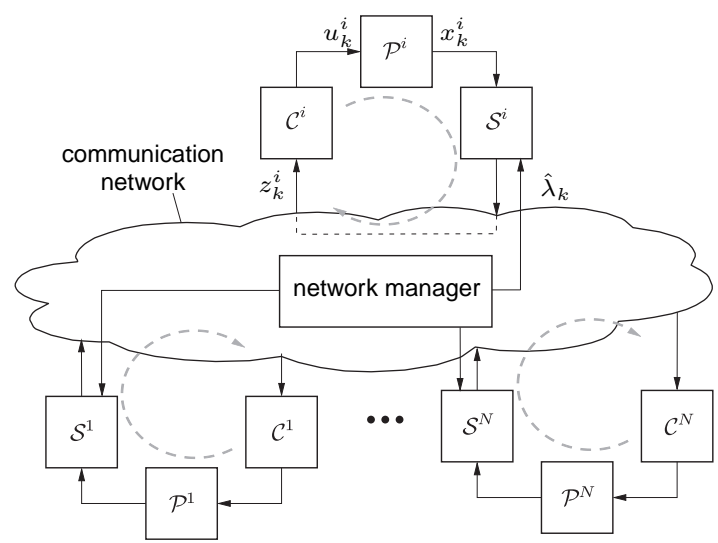

Fig. 1. System model of the networked control system with $N$ control systems closed over a shared communication network with processes $\mathcal{P}^{1}, \ldots, \mathcal{P}^{N}$, sensors $\mathcal{S}^{1}, \ldots, \mathcal{S}^{N}$ and controllers $\mathcal{C}^{1}, \ldots, \mathcal{C}^{N}$. The network manager broadcasts the same variable $\lambda_{k}$ to all subsystems.

rate of the $i$ th subsystem is defined as

$$
r^{i}=\limsup _{T \rightarrow \infty} \frac{1}{T} \mathrm{E}\left[\sum_{k=0}^{T-1} \delta_{k}^{i}\right]
$$

and the total average transmission rate as

$$
y=\sum_{i=1}^{N} r^{i}
$$

The communication constraint is given by limiting the total average transmission rate, i.e.,

$$
y \leq c, \quad c>0
$$

The work in [4] demonstrates that the communication constraint in (5) is remarkably suitable to approximate communication systems with a limited number of transmission slots per time step, although the occurrence of collisions is not modelled in above constraint. In particular, the optimal eventtriggered controller based on this approximation converges to the optimal solution with a limited number of transmissions per time step for increasing number of subsystems. Therefore, the constraint (5) serves as an adequate model.

Each subsystem $i \in\{1, \ldots, N\}$ has a cost function $J^{i}$ given by the linear quadratic average-cost criterion

$$
J^{i}=\limsup _{T \rightarrow \infty} \frac{1}{T} \mathrm{E}\left[\sum_{k=0}^{T-1} x_{k}^{i, \boldsymbol{T}} Q_{x}^{i} x_{k}^{i}+u_{k}^{i, \boldsymbol{T}} Q_{u}^{i} u_{k}^{i}\right] .
$$

The weighting matrix $Q_{x}^{i}$ is positive definite and $Q_{u}^{i}$ is positive semi-definite for each $i \in\{1, \ldots, N\}$. We assume that the pair $\left(A^{i}, B^{i}\right)$ is stabilizable and the pair $\left(A^{i}, Q_{x}^{i, \frac{1}{2}}\right)$ is detectable with $Q_{x}^{i}=\left(Q_{x}^{i, \frac{1}{2}}\right)^{\top} Q_{x}^{i, \frac{1}{2}}$. It is assumed that the sensor and the controller of the $i$ th subsystem merely have knowledge of the local system parameters. These are $A^{i}, B^{i}, W^{i}$, the distribution of $x_{0}$, and $Q_{x}^{i}, Q_{u}^{i}$ of (6).

The control law $\gamma^{i}=\left\{\gamma_{0}^{i}, \gamma_{1}^{i}, \ldots\right\}$ reflecting the behavior of controller $\mathcal{C}^{i}$ is described by causal mappings $\gamma_{k}^{i}$ of the past observations for each time step $k$, i.e.,

$$
u_{k}^{i}=\gamma_{k}^{i}\left(Z_{k}^{i}\right) \text {, }
$$


where $Z_{k}^{i}$ is the observation history until time $k$ of subsystem $i$. We distinguish between two classes of schedulers $\pi^{i}=\left\{\pi_{0}^{i}, \pi_{1}^{i}, \ldots\right\}$ resulting from two types of network managers. In the first case, the network manager broadcasts a fixed parameter $\lambda$ initially and the event-triggered scheduler is then given by

$$
\delta_{k}^{i}=\pi_{k}^{i, \lambda}\left(X_{k}^{i}\right)
$$

where $X_{k}^{i}$ is the state history of subsystem $i$. It should be remarked that we will usually omit $\lambda$ for notational convenience. In the second case, the network parameter $\hat{\lambda}_{k}$ changes over time $k$ and the scheduler adapts its law w.r.t. to $\hat{\lambda}_{k}$, i.e.,

$$
\delta_{k}^{i}=\pi_{k}^{i, \hat{\lambda}_{k}}\left(X_{k}^{i}\right)
$$

The parameter $\hat{\lambda}_{k}$ itself is given by a causal mapping $\mathrm{f}_{k}$ of the past transmission history, i.e.,

$$
\hat{\lambda}_{k}=\mathrm{f}_{k}\left(\delta_{0}^{1}, \ldots, \delta_{0}^{N}, \ldots, \delta_{k}^{1}, \ldots, \delta_{k}^{N}\right)
$$

The mapping $\mathrm{f}_{k}$ represents the adaptation mechanism of the network manager to the current traffic and will be specified later in more detail.

The design objective is to find the optimal control laws $\gamma^{i}$ and optimal scheduling laws $\pi^{i}, i \in\{1, \ldots, N\}$ that minimize the social cost given as an average cost criterion, while satisfying the rate constraint defined in (5). The social cost is defined by the sum of the individual costs $J^{i}$ of each subsystem. Therefore, the optimization problem can be summarized as follows.

$$
\underset{\begin{array}{l}
\gamma^{1}, \ldots, \gamma^{N} \\
\pi^{1}, \ldots, \pi^{N}
\end{array}}{\min } \sum_{i=1}^{N} J^{i} \quad \text { s.t. } y \leq c .
$$

It should be noted that optimization problems with an average cost criterion as given above are underselective [11]. This is because it does not matter how well the policy works at the beginning. Only its stationary behavior determines its cost and the feasibility of the constraint. Therefore, there may exist two policies, which differ completely with respect to their performance in the first $k$ time steps, but eventually converge to the same stationary behavior and therefore yield the same average cost. Hence, the optimization problem does not distinguish between these two policies. For the purpose of our work, we will however consider this feature as an advantage, as it allows us to design an adaptation mechanism for each subsystem that learns the appropriate transmission rate that achieves the optimal performance.

\section{DISTRIBUTED OPTIMIZATION}

In this section, ideas from dual decomposition and adaptive MDPs are used to develop a distributed approach that solve optimization problem (7). In the first part, a Lagrange approach is taken to formulate the dual problem of (7), which enables us to derive a dual price exchange mechanism that broadcasts a price to use the communication network to each subsystem. A corresponding sample-path algorithm is proposed in the second part that estimates the average total transmission rate to approximate the pricing gradient. The implementation of the algorithm is discussed in the last part.

\section{A. Lagrange approach}

In the following, we define the Lagrangian function, introduced in [12] for constrained MDPs, by

$$
\mathcal{L}\left(\pi^{1}, \ldots, \pi^{N}, \gamma^{1}, \ldots, \gamma^{N}, \lambda\right)=\sum_{i=1}^{N} J^{i}+\lambda(y-c)
$$

With this, we can rewrite the optimization problem given by (7) into the corresponding dual problem

$$
\begin{array}{cc}
\max _{\lambda \geq 0} & \min _{\substack{\gamma^{1}, \ldots, \gamma^{N} \\
\pi^{1}, \ldots, \pi^{N}}} \mathcal{L}\left(\pi^{1}, \ldots, \pi^{N}, \gamma^{1}, \ldots, \gamma^{N}, \lambda\right) .
\end{array}
$$

The Lagrange multiplier $\lambda$ can be interpreted as a penalty or price for the transmission rate. Therefore, we sometimes refer to $\lambda$ as the communication penalty or price. By reordering the terms in $\mathcal{L}$ and using the definition of $y$ in (4), we obtain

$$
\mathcal{L}(\cdot, \lambda)=\sum_{i=1}^{N}\left(J^{i}+\lambda r^{i}\right)-\lambda c .
$$

For a fixed $\lambda \geq 0$ and each subsystem $i$, the values of $J^{i}$ and $r^{i}$ only depend on the choice of the local control law $\gamma^{i}$ and the local scheduling law $\pi^{i}$. Therefore, the minimization in (8) can be stated as a separate local minimization within each subsystem for a fixed $\lambda$, i.e.,

$$
\min _{\gamma^{1}, \pi^{1}}\left(J^{1}+\lambda r^{1}\right)+\cdots+\min _{\gamma^{N}, \pi^{N}}\left(J^{N}+\lambda r^{N}\right) .
$$

From above formulation, we observe that the task of the network manager is to broadcast the price $\lambda$ in order to coordinate the local optimization problems.

Subsequently, a characterization of the local minimization is presented. The above local optimization can be considered as the joint design of control and scheduling for a single-loop system with a communication penalty in the feedback loop. This problem has been studied in various works [13]-[16]. It is shown in [15] that the certainty equivalence controller is optimal. Therefore, the control law is given by

$$
u_{k}^{i}=\gamma_{k}^{i, *}\left(Z_{k}^{i}\right)=-L^{i} \mathrm{E}\left[x_{k}^{i} \mid Z_{k}^{i}\right],
$$

where $L^{i}=\left(B^{i, \mathrm{~T}} P^{i} B^{i}+Q_{u}^{i}\right)^{-1} B^{i, \mathrm{\top}} P^{i} A^{i}$ and $P^{i}$ is the solution of the algebraic Riccati equation

$P^{i}=A^{i, \top}\left(P^{i}-P^{i} B^{i}\left(B^{i, \top} P^{i} B^{i}+Q_{u}^{i}\right)^{-1} B^{i, \top} P^{i}\right) A^{i}+Q_{x}^{i}$.

The reason for this structural property of the optimal control law is mainly given by the nestedness property of the information pattern. The information pattern is nested, since the information available at the controller is a subset of the information available at the scheduler. Furthermore, the work in [14] proves that symmetric scheduling laws for first-order systems are optimal. It is conjectured that this also holds for higher-order systems in the following. It is remarked that if this conjecture fails, the optimal estimator must be extended 
by an additive bias term whose calculation is given in [16]. The optimal estimator can be written as

$$
\mathrm{E}\left[x_{k}^{i} \mid Z_{k}^{i}\right]= \begin{cases}x_{k}^{i} & \delta_{k}^{i}=1 \\ \left(A^{i}-B^{i} L^{i}\right) \mathrm{E}\left[x_{k-1}^{i} \mid Z_{k-1}^{i}\right] & \delta_{k}^{i}=0\end{cases}
$$

with $\mathrm{E}\left[x_{0}^{i} \mid Z_{0}^{i}\right]=\mathrm{E}\left[x_{0}^{i}\right]$ for $\delta_{0}^{i}=0$. It should be noted that the optimal control law is independent of $\lambda$. Therefore, the control law can be fully implemented prior to execution without additional knowledge.

By defining the estimation error

$$
e_{k}^{i}=x_{k}^{i}-\mathrm{E}\left[x_{k}^{i} \mid Z_{k-1}^{i}\right],
$$

the remaining problem to determine the optimal scheduling law $\pi^{i, \lambda}$ can be cast as the following MDP with state $e_{k}^{i}$

$$
\min _{\pi^{i, \lambda}} \lim _{T \rightarrow \infty} \frac{1}{T} \mathrm{E}\left[\sum_{k=0}^{T-1}\left(1-\delta_{k}^{i}\right) e_{k}^{i, \mathrm{~T}} Q_{e}^{i} e_{k}^{i}+\lambda \delta_{k}^{i}\right]+\operatorname{tr}\left[P^{i} W^{i}\right],
$$

where $Q_{e}^{i}=L^{i, \mathrm{~T}}\left(Q_{u}^{i}+B^{i, \mathrm{~T}} P^{i} B^{i}\right) L^{i}$ and $e_{k}^{i}$ is described as a $\delta_{k}^{i}$-controlled Markov chain evolving by the following difference equation

$$
e_{k+1}=\left(1-\delta_{k}\right) A e_{k}+w_{k}
$$

with initial condition $e_{0}=x_{0}-\mathrm{E}\left[x_{0}\right]$. The additional term $\operatorname{tr}\left[P^{i} W^{i}\right]$ is constant and can be omitted from the optimization in (10). Optimal policies are therefore stationary mappings of the estimation error $e_{k}^{i}$. Under some mild conditions on the admissible policies $\pi^{i, \lambda}$ given in [13], above optimization problem can be solved by value iteration [11].

It can be observed that the resulting optimal scheduling law is a symmetric threshold policy and takes the following form for a first-order subsystem

$$
\delta_{k}^{i}=\pi^{i, \lambda}\left(e_{k}\right)=\mathbb{1}_{\left\{\left|e_{k}^{i}\right|>d^{i}(\lambda)\right\}}
$$

parameterized by the threshold $d^{i}$ that depends on the price $\lambda$. By varying $\lambda \in(0, \infty)$ in (10) different pairs of individual costs $J^{i}$ and transmission rates $r^{i}$ are attained by the optimal $\pi^{i, \lambda, *}$ for each subsystem $i \in\{1, \ldots, N\}$. In fact, it has been shown in [4] that the relation between optimal $J^{i}$ and $r^{i} \in(0,1)$ is described by a decreasing and strictly convex function, which is denoted by $J^{i}\left(r^{i}\right)$ in the following. Subsequently, we also assume that $J^{i}\left(r^{i}\right)$ is twice continuously differentiable. Then, the optimization in (8) can be rewritten as the dual formulation of a network utility maximization problem [10] with a single link, i.e.,

$$
\max _{\lambda \geq 0} \min _{r^{i}, i \in\{1, \ldots, N\}} \sum_{i=1}^{N} J^{i}\left(r^{i}\right)+\lambda(y-c) .
$$

It is well known that this problem has a unique solution for assigning the optimal transmission rates $r^{i}, i \in\{1, \ldots, N\}$. As there is only one link, it is also clear that the resource constraint $y \leq c$ will be fulfilled with equality. Let $g(\lambda)$ be defined as

$$
g(\lambda)=\min _{r^{i}, i \in\{1, \ldots, N\}} \sum_{i=1}^{N} J^{i}\left(r^{i}\right)+\lambda(y-c) .
$$

In [10], it is shown that the derivative of $g(\lambda)$ with respect to $\lambda$ is obtained by

$$
\frac{\partial g(\lambda)}{\partial \lambda}=y-c
$$

Therefore, the projected gradient algorithm for the dual problem is given by

$$
\lambda_{k+1}=\left[\lambda_{k}+\beta_{k}\left(y_{k}-c\right)\right]^{+}
$$

with an arbitrary initial value $\lambda_{0}$ and $\beta_{k}>0$ for all $k$. The projection is needed to guarantee that the penalty $\lambda_{k}$ remains non-negative at all times $k$. The total transmission rate is defined as

$$
y_{k}=\sum_{i=1}^{N} r_{k}^{i}
$$

where $r_{k}^{i}$ is the average transmission rate defined in (3) assuming that the controller $\gamma^{i}$ given by (9) and the scheduling law $\pi^{i, \lambda_{k}, *}$ obtained from (10) are used. The complete algorithm can be regarded as a dual price exchange mechanism: after broadcasting the price $\lambda_{k}$ by a central network manager to all subsystems at time step $k$, each subsystem adjusts its scheduling policy according to the local optimization problem (10) with $\lambda_{k}$ as the dual price. It is shown in [17] that the algorithm converges to the optimal price $\lambda^{*}$ for sufficiently small $\beta_{k}$.

Above gradient method is completely decoupled from the actual dynamics of the subsystems. Hence, the optimal price $\lambda^{*}$ can be calculated prior to execution and is spread to the subsystems that use the stationary eventtrigger $\delta_{k}^{i}=\pi^{i, \lambda^{*}, *}\left(e_{k}^{i}\right)$ for $k \geq 0$.

\section{B. Adaptive sample-based Algorithm}

The drawback of the gradient method in (14) is obvious. The total average transmission rate $y_{k}$ is not exactly known at time step $k$, as the central network manager would have needed to gather information about every individual transmission rate $r^{i}$ from each subsystem, which also is defined in the limit $T \rightarrow \infty$. Instead, we consider an estimate $\hat{y}_{k}$ of the total transmission rate to approximate the gradient in (14). Therefore, the mapping $\mathrm{f}_{k}$ representing the network manager introduced in Section II is determined by

$$
\hat{\lambda}_{k+1}=\left[\hat{\lambda}_{k}+\beta_{k}\left(\hat{y}_{k}-c\right)\right]^{+} .
$$

The estimate of $y_{k}$ is performed at the network manager by using a moving window technique taking the $T_{\mathrm{W}}$ most recent measurements, i.e.,

$$
\hat{y}_{k}=\frac{1}{T_{\mathrm{W}}} \sum_{\ell=\left[k-T_{\mathrm{W}}+1\right]}^{k} \sum_{i=1}^{N} \delta_{\ell}^{i} .
$$

A less memory consuming implementation of the estimate is given by the recursive formulae

$$
\begin{aligned}
\hat{y}_{k+1} & =\hat{y}_{k}+\frac{1}{T_{\mathrm{W}}} \sum_{i=1}^{N}\left(\delta_{k+1}^{i}-\delta_{k-T_{\mathrm{w}}+1}^{i}\right) \\
& =h_{T_{\mathrm{w}}}\left(\hat{y}_{k}, \delta_{k+1}^{1}, \delta_{k-T_{\mathrm{w}}+1}^{1}, \ldots, \delta_{k+1}^{N}, \delta_{k-T_{\mathrm{w}}+1}^{N}\right),
\end{aligned}
$$


where it is assumed that no transmission occurs at times before 0 , i.e., $\delta_{k}^{i}=0$ for all $k<0$. By choosing the step size $\beta_{k}$ to be

$$
\beta_{k}= \begin{cases}\frac{T_{\mathrm{W}}}{k} & k \in\left\{m T_{\mathrm{W}} \mid m \in \mathbb{Z}^{+}\right\} \\ 0 & \text { otherwise }\end{cases}
$$

and using the fact that the solution $\pi^{i, \lambda}$ for an arbitrary $\lambda$ yields an ergodic Markov chain, i.e.,

$$
\lim _{T_{\mathrm{W}} \rightarrow \infty} \hat{y}_{T_{\mathrm{W}}}=y_{T_{\mathrm{W}}}, \quad \mathrm{P} \text {-a.s., }
$$

we observe that the sample-path algorithm in (15) is a good approximation of (14) for a large window size $T_{\mathrm{W}}$. The drawback of choosing the step size as defined above is slow convergence. Therefore, we will consider a step size of $\frac{1}{k}$ in the following numerical simulations. This choice is very common for recursive algorithms, where the gradient is noisy as $\sum_{k=0}^{\infty} \beta_{k}=\infty$ and $\sum_{k=0}^{\infty} \beta_{k}^{2}<\infty$, see, e.g., [18].

Figure 2 summarizes the mechanism of the complete adaptive event-triggered control system by illustrating one particular subsystem and its interplay with the network manager. In contrast to the design mechanism described at the end of subsection III-A, the price is not determined prior to execution, but is continuously estimated within the network manager for every time step $k$.

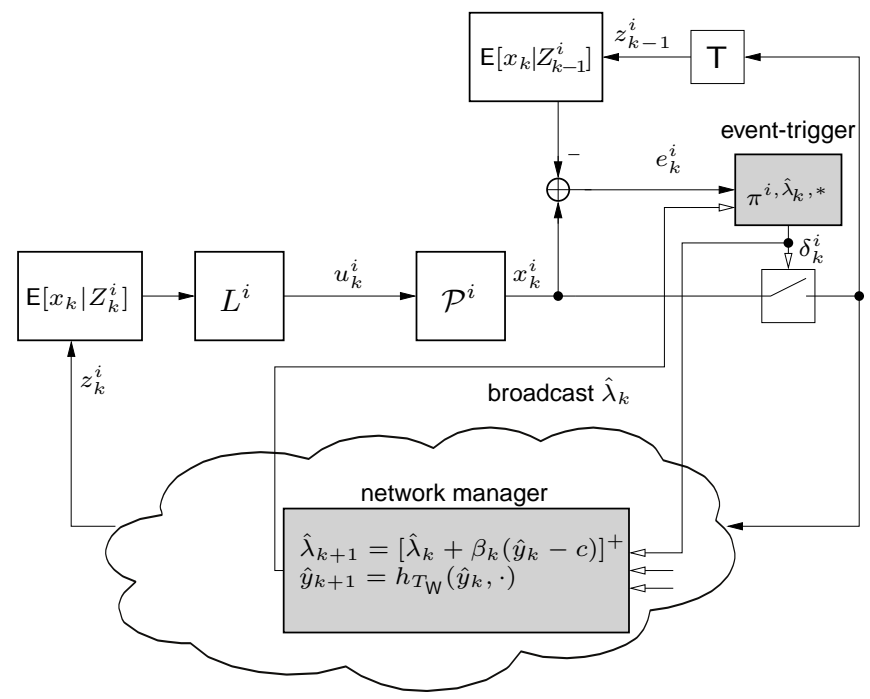

Fig. 2. Complete structure of the networked control for subsystem $i$ with optimal event-triggered scheduler $\pi^{i, \hat{\lambda}_{k}, *}$ that adapts its law according to the price $\hat{\lambda}_{k}$. The price $\hat{\lambda}_{k}$ is broadcasted to each subsystem from the central network manager. System block T denotes a 1-step delay element.

\section{Implementation and Discussion}

The implementation of the overall system is accomplished in two phases. In the first phase, which is performed before execution and locally in each subsystem, the optimal controller $\gamma^{i}$ given by (9) is calculated and the mapping from price $\lambda$ to the optimal event-trigger $\pi^{i, \lambda, *}$ by solving (10) through value iteration. In the second phase, the network manager adjusts the price accordingly to the total average transmission rate estimated given by $\hat{y}_{k}$, which serves as an estimate to approximate the gradient $\frac{\partial g}{\partial \lambda}$. Unlike broadcasting the price $\hat{\lambda}_{k}$ by a network manager, a complete decentralized adaptation mechanism can be realized when each subsystem is able to sense the amount of traffic directly. Then, the calculation of $\hat{\lambda}_{k}$ can be performed locally in each subsystem. In contrast to a time-triggered scheduling mechanism, which needs a global exhaustive search at runtime to find the optimal scheduling sequence, the event-triggered scheme allows therefore a tractable implementation. Apart from the fact that the adaptation mechanism enables the distributed architecture, the local event-triggers are capable to adjust their thresholds according to runtime changes that are often found in real applications. These are for example given by adding or removing control loops during runtime, changes in the resource constraint, or changes in the local system parameters.

\section{NUMERICAL RESULTS}

Suppose the system comprises of two subsystems with system parameters $A^{1}=1.25, B^{1}=1, Q_{x}^{1}=1, Q_{u}^{1}=0$ and $A^{2}=0.75, B^{2}=1, Q_{x}^{2}=1, Q_{u}^{2}=0$, respectively. Both systems start with $x_{0}^{1}=x_{0}^{2}=0$ and the system noise is given by $w_{k} \sim \mathcal{N}(0,1)$. The communication constraint is set to $c=1$. The optimal control gain in (9) is given by $L^{i}=A^{i}$ for each subsystem $i \in\{1,2\}$, which corresponds to a deadbeat control strategy. The optimal scheduling laws for a fixed $\lambda$ of each subsystem is a symmetric threshold policy defined in (12) with threshold $d^{i}, i \in\{1,2\}$. The optimal threshold for various fixed Lagrange multipliers $\lambda$ is obtained by value iteration for the average cost problem in (10). Figure 3 shows the mapping from $\lambda$ to the optimal thresholds of both subsystems. It should be noted that the determination of the mapping shown in Fig. 3 can be performed offline and locally for each subsystem. Therefore, the computationally intensive part for the determination of the optimal threshold can be accomplished before runtime. Figures 4 and 5 show

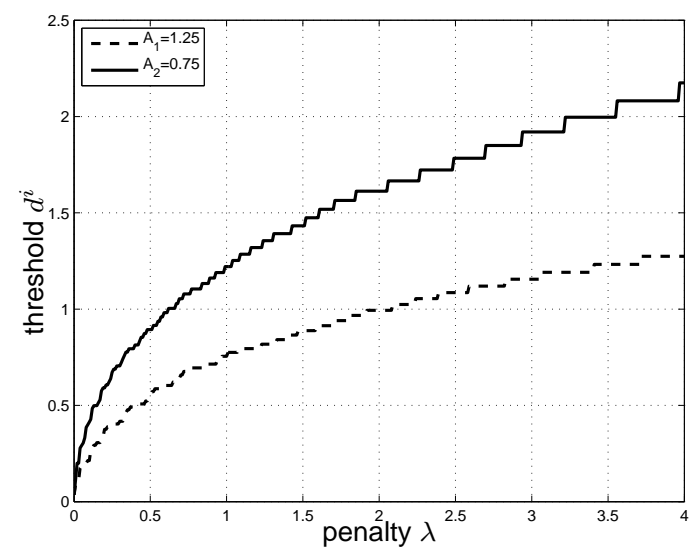

Fig. 3. Mapping from communication penalty $\lambda$ to the optimal symmetric scheduling law described by the threshold $d^{i}$ for subsystem $i, i \in\{1,2\}$.

the sample-path behavior of the adaptive algorithm for a time-horizon of 10000 for the thresholds $d^{i}, i \in\{1,2\}$, communication penalty $\lambda$, and the estimated total transmission 
rate $\hat{y}_{k}$, respectively. The initial state $\hat{\lambda}_{0}$ is set to 1 . The step size $\beta_{k}$ of the gradient algorithm in (15) is chosen to be $\frac{1}{k}$. For the calculation of the estimate $\hat{y}_{k}$ of the total rate, we select a window of $T_{\mathrm{W}}=500$.

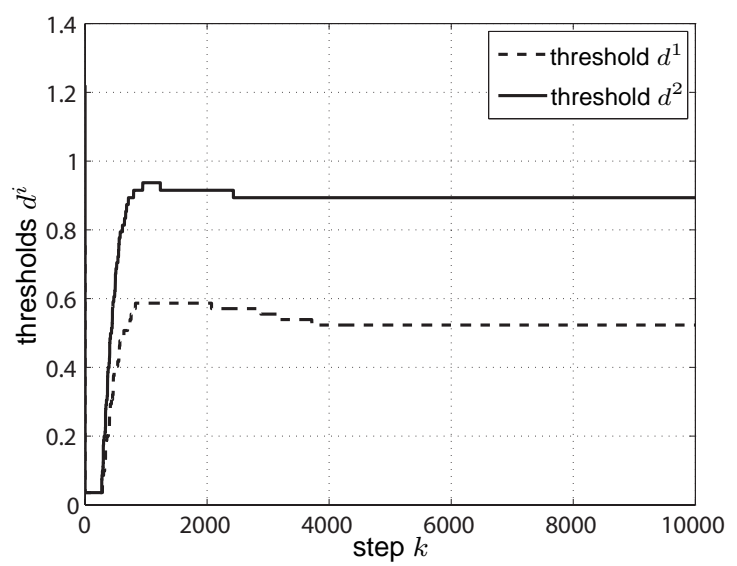

Fig. 4. Sample path of the thresholds $d^{1}$ and $d^{2}$ of the subsystem 1 and 2 .

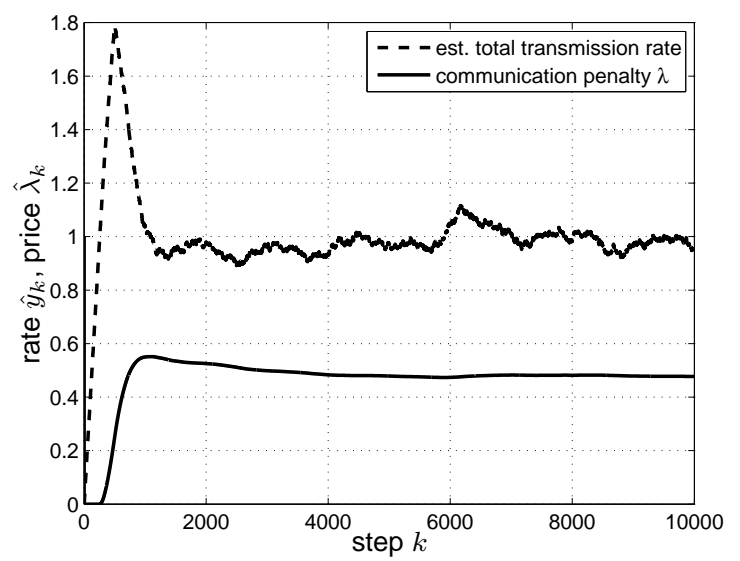

Fig. 5. Sample path of the estimated total transmission rate $\hat{y}_{k}$ and of the communication penalty $\hat{\lambda}_{k}$.

The optimal thresholds have been determined in [4] by solving the global resource allocation problem and are given by $d^{1, *}=0.5$ and $d^{2, *}=0.95$. From Fig. 4 we observe that these optimal are asymptotically attained by the samplepath based algorithm. The communication penalty converges to $\lambda=0.5$, while the total rate converges towards 1 . After approximately 2500 steps convergence is achieved, after which the signal $\hat{y}_{k}$ has a mean of 0.997 and variance 0.0015 . The slow ramp up in the starting phase is due to the fact that $\hat{y}_{k}$ is initialized with 0 . The convergent behavior can be enhanced by choosing a smaller window $T_{\mathrm{W}}$ for the transmission rate estimator leading to a greater variance of the estimate, which implies fluctuations in the thresholds $d^{1}$ and $d^{2}$.

\section{CONCLUSIONS AND OUTLOOK}

Based on a dual price exchange mechanism, this paper develops a framework for the design of distributed eventtriggered control systems that share a common resource. Therein, it is shown that convexity properties of the optimal event-trigger design enable the application of dual formulations related to network utility maximization. The distributed approach is realized by an adaptive event-trigger that adjusts its threshold according to the estimated price for the resource.

The proposed framework opens up a diversity of problems that have to be addressed in the future. A convergence analysis of the overall adaptive system based on stochastic approximation is to be carried out. The consideration of hard communication constraints rather than an average rate constraint with a limited number of transmission slots is another line of research to be tackled in the future.

\section{REFERENCES}

[1] K. Åström and B. Bernhardsson, "Comparison of Riemann and Lebesgue sampling for first order stochastic systems," in Proc. 41th IEEE Conference on Decision and Control (CDC'02), 2002.

[2] T. Henningsson and A. Cervin, "Scheduling of event-triggered controllers on a shared network," in Proc. 47th IEEE Conference on Decision and Control, (Cancun, Mexico), 2008.

[3] J. Lunze and D. Lehmann, "A state-feedback approach to event-based control," Automatica, vol. 46, no. 1, pp. 211-215, 2010.

[4] A. Molin and S. Hirche, "On the optimal design of decentralized event-triggered controllers for large-scale systems with contentionbased communication," in Proc. 50th IEEE Conference on Decision and Control (CDC'11), 2011.

[5] D. Dimarogonas, E. Frazzoli, and K. Johansson, "Distributed eventtriggered control for multi-agent systems," Automatic Control, IEEE Transactions on, vol. 57, pp. 1291 -1297, may 2012.

[6] P. Tabuada, "Event-triggered real-time scheduling of stabilizing control tasks," Automatic Control, IEEE Transactions on, vol. 52, no. 9, pp. $1680-1685,2007$.

[7] R. Blind and F. Allgöwer, "Analysis of Networked Event-Based Control with a Shared Communication Medium: Part I - Pure ALOHA," in IFAC World Congress, 2011.

[8] R. Blind and F. Allgöwer, "Analysis of Networked Event-Based Control with a Shared Communication Medium: Part II - Slotted ALOHA," in IFAC World Congress, 2011.

[9] R. Blind and F. Allgöwer, "On the optimal sending rate for networked control systems with a shared communication medium," in Proc. 50th IEEE Conference on Decision and Control (CDC'11), 2011.

[10] S. Shakkottai and R. Srikant, "Network optimization and control," Foundations and Trends in Networking, vol. 2, no. 3, pp. 271-379, 2007.

[11] O. Hernández-Lerma, Adaptive Markov control processes, vol. 79. Springer New York, 1989.

[12] E. Altman, Constrained Markov Decision Processes. Chapman and Hall/CRC, 1999.

[13] Y. Xu and J. Hespanha, "Optimal communication logics in networked control systems," Decision and Control, 2004. CDC. 43rd IEEE Conference on, vol. 4, pp. 3527-3532 Vol.4, Dec. 2004.

[14] G. Lipsa and N. Martins, "Remote state estimation with communication costs for first-order LTI systems," Automatic Control, IEEE Transactions on, vol. 56, no. 9, pp. 2013-2025, 2011.

[15] A. Molin and S. Hirche, "On LQG joint optimal scheduling and control under communication constraints," in Proc. 48th IEEE Conference on Decision and Control, 2009.

[16] A. Molin and S. Hirche, "Structural characterization of optimal eventbased controllers for linear stochastic systems," in Proc. 49th IEEE Conference on Decision and Control (CDC'10), 2010.

[17] S. Low and D. Lapsley, "Optimization flow control, I: basic algorithm and convergence," Networking, IEEE/ACM Transactions on, vol. 7, no. 6, pp. 861-874, 1999.

[18] X. Cao, Stochastic learning and optimization: a sensitivity-based approach, vol. 17. Springer-Verlag New York Inc, 2007. 